\title{
Race/Ethnicity and 30-Day Readmission Rates in Medicare Beneficiaries With COPD
}

\author{
Daneen R Nastars, Josè D Rojas, Kenneth J Ottenbacher, and James E Graham
}

\begin{abstract}
BACKGROUND: COPD is now included in Medicare's hospital readmission reduction program. Hospitals with excessive risk-adjusted 30-d readmission rates receive financial penalties. Race/ethnicity is not included in the risk-adjustment models. We examined whether race/ethnicity was independently associated with readmission after controlling for clinical factors and other demographic variables. METHODS: We used the $\mathbf{1 0 0 \%}$ Medicare in-patient (Part A) files to identify patients hospitalized with COPD (MS-DRG codes 190, 191, 192) who were discharged between January 1, 2013, and September 13, 2014. The outcome measure was an unplanned readmission within $30 \mathrm{~d}$ of hospital discharge. We used generalized linear mixed models to test the independent effects of race/ethnicity on 30-d readmission. RESULTS: The sample included 298,706 Medicare beneficiaries hospitalized for COPD: 87\% white, 8\% African-American, and 5\% Hispanic. Mean age was $77.7 \pm 7.7 \mathrm{y}$. Overall, $17.3 \%$ of subjects experienced an unplanned readmission. Whites $(\mathbf{1 7 . 4 \% )}$ and African-Americans (17.7\%) had significantly higher unadjusted rates than Hispanics, and Hispanics demonstrated the lowest readmission rate $(16.3 \%)$. The minority groups generally displayed higher-risk clinical profiles. After controlling for those differences, the multivariable model suggested a benefit for both minority groups in terms of readmission risk. The adjusted readmission rates for whites, African-Americans, and Hispanics were 16.6\%, 15.9\%, and 14.6\%, respectively. CONCLUSIONS: Racial/ethnic disparities in observed readmission rates may be largely explained by the more severe clinical profiles of minority populations. Controlling for known clinical risk factors effectively mediates the relationship between race/ethnicity and readmission. Key words: COPD; ethnic groups; healthcare reform; hospitalization; 30-day readmission. [Respir Care 2019;64(8):931-936. () 2019 Daedalus Enterprises]
\end{abstract}

\section{Introduction}

The Medicare Hospital Readmissions Reduction Program (HRRP), developed in response to the Affordable Care Act, was implemented on October 1, 2012 (https://www.cms.gov/ Medicare/Medicare-Fee-for-Service-Payment/AcuteInpatient

\footnotetext{
Ms Nastars and Dr Rojas are affiliated with the Department of Respiratory Care, University of Texas Medical Branch, Galveston, Texas. Dr Ottenbacher is affiliated with the Division of Rehabilitation Sciences, University of Texas Medical Branch, Galveston, Texas. Dr Graham is affiliated with the Department of Occupational Therapy, Colorado State University, Fort Collins, Colorado.
}

Ms Nastars presented a version of this paper at the AARC Congress, held October 4-7, 2017, in Indianapolis, Indiana.

The authors disclose a relationship with the National Institutes of Health (grants P2C-HD065702, R01-HD069443).
PPS/Readmissions-Reduction-Program.html, Accessed June 21, 2018). Under the HRRP, hospitals with higher-than-expected 30-d readmission rates for select medical conditions receive financial penalties from the Centers for Medicare and Medicaid Services (CMS). COPD was added to the list of eligible conditions in fiscal year 2015.

The Affordable Care Act has improved access to health insurance, but there are still obstacles, such as health literacy, patient beliefs, access to health care centers, language barriers, limited cultural competency by health care providers, and lack of appropriate clinical guidelines. ${ }^{1}$ Prior studies have shown racial disparities in overall hospital

Correspondence: Daneen R Nastars MSc RRT RRT-ACCS, 301 University Blvd, Galveston, Texas, 77555. E-mail: danastar@utmb.edu

DOI: $10.4187 /$ respcare. 06475 
care and in the treatment of COPD. ${ }^{2}$ It is also worth noting that the death rate for patients with COPD has risen more for African-Americans than whites and for women than men. ${ }^{2}$

In the first year of the HRRP, high penalties were disproportionately allotted to hospitals that predominantly serve the indigent population. ${ }^{3-5}$ To lower readmission rates, some hospitals have implemented programs prior to discharge to educate patients about medications, disease, action plan, nutrition, smoking cessation, and counseling. ${ }^{5}$ Most of the studies conducted for reducing COPD readmission rates are focused on interventions after discharge. ${ }^{6}$ Examples of post-discharge care include patient followup, pulmonary rehabilitation, and telehealth. ${ }^{5}$ One study identified predictors of hospital readmissions as comorbidities, improper respiratory drug use, and lack of health care utilization. ${ }^{7}$

To address some of CMS released the issues raised in these studies, in 2015 a guide to preventing readmissions among racially and ethnically diverse Medicare beneficiaries. ${ }^{8}$ This guide addresses the increasingly diverse patient populations and outlines strategies to prevent readmissions in minority patients. Our primary objective was to determine whether race/ethnicity is independently associated with risk for readmission after controlling for other demographic characteristics and differences in clinical profiles between racial/ethnic groups.

\section{Methods}

\section{Data Source}

Data were obtained from Medicare in-patient (Part A) claims from 2012 to 2014. The Beneficiary Summary File included patient demographics and eligibility information. The Medicare Provider and Analysis Record (MedPAR) included claims information from all in-patient admissions for Medicare fee-for-service beneficiaries. The University of Texas Medical Branch institutional review board approved the study, and there was an agreement from the CMS to use the data.

\section{Sample Selection}

We identified patients hospitalized for COPD according to Medicare Severity Diagnosis-Related Groups (MSDRG) codes 190, 191, or 192. These patients were discharged between January 1, 2013, and September 30, 2014. We also collected data from 2012 to gain information on previous admissions 12 months before the index hospitalizations in 2013. If patients had > 1 COPD hospitalization during the study period, we selected the first as the index stay. The original sample included 558,239 patients. We excluded patients who died during the index hospitaliza-

\section{QUICK LOOK}

\section{Current knowledge}

The addition of COPD as a medical condition eligible for financial penalty as a result of higher-than-expected 30-d readmission rates is a significant concern for respiratory care departments. Health disparities by race and ethnic background have been reported.

\section{What this paper contributes to our knowledge}

A survey of Medicare data sets for unplanned 30-d COPD readmission revealed differences in readmission rates by race and ethnic origin. When controlling for demographics and prior health care utilization, whites had a higher readmission rate. This work demonstrates a need for more research on the impact of various factors that can improve outcomes for COPD patients.

tion or within $30 \mathrm{~d}$ of discharge $(n=15,799)$, were $<66 \mathrm{y}$ old at admission $(n=115,219)$, were not classified as white, African-American, or Hispanic $(n=10,477)$, and who enrolled in a Medicare health maintenance organization during the 12 months before or within $30 \mathrm{~d}$ after their index hospitalization $(n=118,068)$. The final sample included 298,676 subjects.

\section{Outcome Variable}

We examined 30-d unplanned hospital readmissions, which were identified as a claim for an unplanned acute hospital stay in the MedPAR file within in $30 \mathrm{~d}$ of a previous discharge. Planned readmissions were not counted as described in the 2017 All-Cause Hospital-Wide Measure Updates and Specifications Report. ${ }^{9}$

\section{Independent Variable}

Race/ethnicity classification was taken from the RTI International race variable in the Beneficiary Summary File. We limited the categories to white, African-American, and Hispanic, with white being used as the reference group for the multivariable analyses.

\section{Covariates}

Basic demographic information included age and sex. We used disability status to indicate whether the subject was eligible for Medicare coverage initially due to disability. The primary diagnosis was based on the MS-DRG code for admission (190: COPD with major complications and comorbidities (MCC); 191: COPD with complications 


\section{Race/Ethnicity and 30-Day ReAdMission Rates}

Table 1. Sample Characteristics Overall and Stratified by Race/Ethnicity

\begin{tabular}{|c|c|c|c|c|c|}
\hline \multirow{2}{*}{ Characteristics } & \multirow{2}{*}{ Total } & \multicolumn{3}{|c|}{ Race/Ethnicity } & \multirow{2}{*}{$P^{*}$} \\
\hline & & White & African-American & Hispanic & \\
\hline Subjects, $n$ & 298,706 & 258,864 & 25,052 & 14,790 & \\
\hline Age, y, mean (SD) & 77.7 (7.7) & $77.8(7.7)$ & $76.3(7.6)$ & $78.5(7.9)$ & $<.001$ \\
\hline Female, $\%$ & 59.6 & 59.5 & 60.5 & 60.3 & $<.001$ \\
\hline Disability, \% & 20.9 & 20.0 & 30.5 & 20.0 & $<.001$ \\
\hline MS-DRG, \% & & & & & $<.001$ \\
\hline 190 & 41.1 & 41.8 & 36.1 & 37.8 & \\
\hline 191 & 35.2 & 34.8 & 38.7 & 35.5 & \\
\hline 192 & 23.7 & 23.4 & 25.1 & 26.7 & \\
\hline Elixhauser comorbidities, $\%$ & & & & & $<.001$ \\
\hline $0-2$ & 17.0 & 17.4 & 13.5 & 16.6 & \\
\hline $3-4$ & 40.1 & 40.1 & 40.2 & 40.6 & \\
\hline $5+$ & 42.9 & 42.5 & 46.3 & 42.8 & \\
\hline Length of stay, d, mean (SD) & $4.2(2.9)$ & $4.2(2.9)$ & $4.3(3.0)$ & $4.6(3.2)$ & $<.001$ \\
\hline ICU admission, \% & 22.4 & 22.1 & 23.1 & 25.7 & $<.001$ \\
\hline Prior acute stays, $n$, mean (SD) & $0.9(1.4)$ & $0.9(1.4)$ & $1.1(1.6)$ & $1.0(1.5)$ & $<.001$ \\
\hline 30-d readmission, \% & $17.3 \%$ & $17.4 \%$ & $17.7 \%$ & $16.3 \%$ & $<.001$ \\
\hline \multicolumn{6}{|c|}{$\begin{array}{l}\text { Medicare patient demographics of patients with COPD discharged between January 1, 2013, and September 30, 2014. Prior acute stays based on hospital admissions } 12 \text { months prior to January } 1 \text {, } \\
\text { 2013. } \\
\text { MS-DRG = Medicare Severity Diagnosis-Related Group }\end{array}$} \\
\hline
\end{tabular}

and comorbidities (CC); and DRG 192: COPD without any $\mathrm{MCC} / \mathrm{CC}$ ). A comorbidity measure was calculated as the sum of conditions a subject had from the 30-item Elixhauser Comorbidity Index. ${ }^{10}$ The summed score was recoded into 3 categories: $0-2,3-4$, or 5+ comorbidities. Length of stay was calculated in days. Admission to an ICU during the index hospital stay was recorded as a yes or no variable. As a final covariate, we calculated the number of previous hospital admissions over the previous year.

\section{Data Analysis}

Descriptive summaries for all subject demographic and clinical characteristics as well as the 30-d readmission variable were calculated and stratified by race/ethnicity. Differences between race/ethnicity groups were tested using analysis of variance and chi-square tests for numerical and categorical variables, respectively. We then used a series of 3 multilevel logistic regression models test the independent effects of race/ethnicity on risk of unplanned 30-d readmission. Specifically, we used generalized linear mixed models with logit link. The multilevel approach controls for the clustering of patients within hospitals. Model 1 included demographic variables only: age, sex, and race/ethnicity. Model 2 included the demographic variables along with 5 clinical variables: disability, primary diagnosis, comorbidity burden, length of stay, and ICU admission. Model 3 included all variables from Model 2 plus the number of acute admissions over the previous year.
Adjusted probabilities from all 3 multivariable models were then calculated and plotted. The SPSS v24 software (IBM, Armonk, New York) was used for all analyses.

\section{Results}

The overall subject demographics and profiles for all 3 race/ethnicity groups are included in Table 1. Of the 298,706 subjects, 86.6\% were white, $8.3 \%$ were AfricanAmerican, and $4.9 \%$ were Hispanic. There were statistically significant differences $(P<.001)$ between the race/ethnicity groups in all covariates and the outcome. Hispanics were older, on average, than the other 2 groups. African-Americans had a slightly higher percentage of women and substantially higher percentages of disability compared to whites and Hispanics. Whites had the highest percentage of major complications with comorbidities, which was the most severe DRG (190), but African-Americans had the highest percentage with $5+$ comorbidities from the Elixhauser Comorbidity Index. Hispanics experienced longer lengths of stay and were more likely to have been in the ICU during their index hospital admission, whereas African-Americans had more prior hospital admissions than the other 2 groups. African-Americans demonstrated the highest unadjusted 30 -d readmission rates $(17.7 \%)$, whereas whites exhibited a slightly lower $17.4 \%$, and Hispanics demonstrated the lowest readmission rate $(16.3 \%)$.

Results from the 3-step multivariable logistic regression are shown in Table 2. Using whites as the reference group, 


\section{Race/EthNicity and 30-DAy READMISSION RATES}

Table 2. Results From 3-Step Generalized Linear Mixed Regression Models for 30-Day Unplanned Readmissions

\begin{tabular}{|c|c|c|c|}
\hline & Model 1 & Model 2 & Model 3 \\
\hline Age, 5-y intervals & $1.06(1.05-1.06)$ & $1.05(1.04-1.05)$ & $1.05(1.04-1.05)$ \\
\hline Male & $1.10(1.08-1.12)$ & $1.13(1.11-1.15)$ & $1.14(1.11-1.16)$ \\
\hline \multicolumn{4}{|l|}{ Race/ethnicity } \\
\hline White & Reference & Reference & Reference \\
\hline African-American & $1.03(1.00-1.07)$ & $0.99(0.96-1.03)$ & $0.95(0.92-0.98)$ \\
\hline Hispanic & $0.92(0.88-0.96)$ & $0.90(0.86-0.94)$ & $0.86(0.82-0.91)$ \\
\hline Disability & NA & $1.15(1.13-1.18)$ & $1.08(1.05-1.11)$ \\
\hline 192 & NA & Reference & Reference \\
\hline 191 & NA & $1.25(1.21-1.28)$ & $1.20(1.17-1.23)$ \\
\hline 190 & NA & $1.26(1.23-1.30)$ & $1.21(1.17-1.24)$ \\
\hline \multicolumn{4}{|c|}{ Elixhauser comorbidities } \\
\hline $0-2$ & NA & Reference & Reference \\
\hline $3-4$ & NA & $1.21(1.17-1.25)$ & $1.15(1.11-1.18)$ \\
\hline $5+$ & NA & $1.64(1.59-1.69)$ & $1.43(1.38-1.47)$ \\
\hline Length of stay & NA & $1.06(1.05-1.06)$ & $1.05(1.05-1.06)$ \\
\hline ICU admission & NA & $1.11(1.09-1.14)$ & $1.11(1.08-1.14)$ \\
\hline Prior acute stays & NA & NA & $1.26(1.26-1.27)$ \\
\hline
\end{tabular}

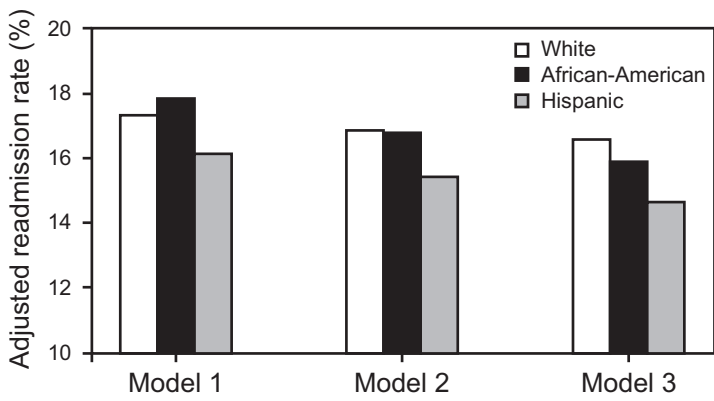

Fig. 1. Adjusted probability for $30-d$ unplanned readmission by race/ethnicity from the multivariable logistic regression models shown in Table 2.

Hispanics showed significantly lower odds of readmission across all 3 models (odds ratio $=0.86,95 \%$ CI $0.82-0.91$ in the final model). African-Americans showed no significant difference compared to whites in the first 2 models; however, after including prior hospital admissions in the model, African-Americans also demonstrated significantly lower odds of readmission (odds ratio $=0.95,95 \%$ CI 0.92-0.98). Adjusted probabilities for 30-d unplanned readmission from the final model, controlling for demographic, clinical, and prior health care utilization, for whites, African-Americans, and Hispanics were 16.6\%, 15.9\%, and $14.6 \%$, respectively (Fig. 1).

\section{Discussion}

COPD is a chronic condition that causes more disabilities, increased comorbidities, and decreased quality of life compared to people without COPD.${ }^{11}$ COPD is primarily caused by smoking, but there has been an increase in incidence in nonsmokers. ${ }^{11}$ Data from the National Health and Nutrition Examination (NHANES III) study indicate that whites have higher rates of air-flow obstruction due to smoking, whereas Hispanics and African-Americans have higher rates of obstruction due to occupational exposure. ${ }^{2}$ Between 1980 and 2000, there was an increase in smoking in the African-American population, and in particular African-American women. The effects of these behaviors are now being seen in this population. ${ }^{2}$ The rate of hospitalizations and emergency department visits for COPD since 1987 has increased, but physician visits have decreased among African-Americans. ${ }^{2}$ Another study showed in a racially integrated, low-income, urban environment that medical mistrust can lead to African-Americans utilizing the emergency department instead of primary care physicians. ${ }^{12}$ Lower socioeconomic status can lead to poor COPD outcomes, and African-Americans had greater disease severity and a higher risk of exacerbation that linked socioeconomic status and other covariates such as comorbidities and occupational exposures. ${ }^{13}$ Some evidence suggests that African-Americans and women are genetically more susceptible to COPD. ${ }^{2}$ African-Americans tend to have lower lung function than whites, as evidenced by lower FVC and $\mathrm{FEV}_{1}$. This is believed to be due to anthropometric differences as well as lower socioeconomic status. ${ }^{2}$ In contrast, Hispanics showed a slower decline in lung function with COPD. ${ }^{14}$ Current American Thoracic Society/European Respiratory Society recommendations for 


\section{Race/Ethnicity and 30-Day Readmission Rates}

performing and reporting spirometry include the suggestion that predicted spirometry reference values be obtained from reference equations derived from a population of similar race/ethnicity if possible. ${ }^{15}$

Many factors can contribute to adverse health outcomes in patients with COPD, including lower education and/or socioeconomic status, lack of health insurance, inadequate housing, and living near environmental hazards. ${ }^{11}$ Minority populations are more susceptible to these risk factors. Comorbid conditions also contribute to poor health outcomes. The percentage of complications, comorbidities, and prior admissions was highest among the AfricanAmerican population and lowest among Hispanics. Studies have shown that African-Americans have lower health literacy and lower medication adherence, and were less likely to follow up with a physician after discharge from the hospital. 3,16 The Institute of Medicine's Report on Unequal Treatment from 2002 stated that there are racial disparities in health care and that they worsen health outcomes. ${ }^{17}$ Our data support the interpretation that health disparities can contribute to poor health outcomes. African-Americans had a greater incidence of disability and a greater incidence of 5+ Elixhauser comorbidities than whites. The existence of multiple comorbidities at patient discharge can have a negative effect on mortality and overall quality of life. ${ }^{13,14,18} \mathrm{~A}$ similar pattern was seen in a study that examined all-cause 30-d readmission rates by race/ethnicity in patients with COPD in an all-payer database from 8 states. ${ }^{19}$ Unadjusted rates for whites, African-Americans, and Hispanics were 19.1\%, 21.5\%, and $19.5 \%$, respectively. ${ }^{19}$

After accounting for differences in clinical profiles and prior hospitalization histories, our results indicate that African-Americans and Hispanics demonstrated significantly lower odds for 30-d readmission compared to whites. Qualitative research is needed to evaluate how culture may be influencing these findings. Shaya et $\mathrm{al}^{20}$ retrospectively analyzed COPD admissions in a Medicaid database and reported that African-American patients utilized fewer medical services and accounted for lower medical costs than did white patients. Singh et al ${ }^{18}$ examined long-term trend data from multiple registries and reported that COPD mortality was lower in African-Americans and Hispanics than in whites. In a prospective cohort study of COPD subjects in an integrated health delivery system, Eisner et $\mathrm{al}^{13}$ reported that African-Americans had greater disease severity and risk of exacerbation than whites and that this difference could be entirely accounted for by differences in socioeconomic status. Differences in multigenerational households, attitudes toward informal caregiving, and mistrust in the medical system could lead to lower utilization of health services by subgroups of the population. Weiss et $\mathrm{al}^{21}$ reported substantial differences in the percentages of older adults who receive informal home care across racial/ethnic groups: $44.3 \%$ Latinos, $33.9 \%$ African-
Americans, and $24.6 \%$ non-Hispanic whites. Care teams should gather information about the amount of informal care elders receive and who is providing that care when determining an individualized treatment plan. ${ }^{21}$ Weiss et $\mathrm{a}^{21}$ believed that gathering information and having no preconceptions can give the care team insight into cultural beliefs of older Latinos and may improve communication. In 2015, CMS released a guide that outlines how hospitals can address key issues that may lead to readmissions and poor health outcomes in racial and ethnic minorities. This is a good start in adapting to diverse populations. The guideline suggests the use of a multidisciplinary team to educate patients on their primary admitting diagnosis as well as any comorbidities during their admission. ${ }^{8} \mathrm{Com}$ prehensive care management programs or integrated disease management interventions are being used for pre- and post-discharge care to help reduce hospital readmissions. ${ }^{5}$ These programs can consist of respiratory therapists, physicians, case managers, and nurses. Werre et al ${ }^{22}$ compared respiratory therapist-driven protocols with physiciandriven protocols and found there was no significant effect on hospital stay between an respiratory therapist-driven and physician-driven protocols, but they suggest that respiratory therapist-driven protocols may help in reducing 30-d readmissions.

Our observational study has some limitations. Coding errors or practices in the hospital claims data may lead to misclassification of the primary and/or comorbid conditions of our cohort. In addition, race/ethnicity is a crude surrogate for social risk factors, and education, socioeconomic status, health behaviors, and environment-related issues vary within race/ethnicity groups. Definitive measures would better reflect the social determinants of health. Finally, the claims data do not have information related to functional status, which is strongly correlated with an individual's overall health and readmission risk.

\section{Conclusion}

Using national Medicare data, we found that, despite worse clinical profiles, minorities with COPD experience comparable 30-d readmissions compared to whites. Controlling for differences in clinical risk factors yielded minority advantages in predicted 30-d unplanned readmission rates. Additional research is needed to better understand the reasons for and consequences of these patterns and how we can improve outcomes for all COPD patients, regardless of their sociodemographic background.

\section{REFERENCES}

1. Celedon JC, Roman J, Schraufnagel DE, Thomas A, Samet J. Respiratory health equality in the United States. The American Thoracic Society perspective. Ann Am Thorac Soc 2014;11(4):473-479. 


\section{Race/Ethnicity and 30-Day ReAdMission Rates}

2. Dransfield MT, Bailey WC. COPD: racial disparities in susceptibility, treatment, and outcomes. Clin Chest Med 2006;27(3):463-471.

3. Chen HF, Homan S, Carlson E, Popoola T, Radhakrishnan K. The impact of race and neighborhood racial composition on preventable readmissions for diabetic Medicare home health beneficiaries. J Racial Ethn Health Disparities 2017;4(4):648-658.

4. Feemster LC, Au DH. Penalizing hospitals for chronic obstructive pulmonary disease readmissions. Am J Respir Crit Care Med 2014; 189(6):634-639.

5. Shah T, Press VG, Huisingh-Scheetz M, White SR. COPD readmissions: addressing COPD in the era of value-based health care. Chest 2016;150(4):916-926.

6. Jennings JH, Thavarajah K, Mendez MP, Eichenhorn M, Kvale P, Yessayan L. Predischarge bundle for patients with acute exacerbations of COPD to reduce readmissions and ED visits: a randomized controlled trial. Chest 2015;147(5):1227-1234.

7. Baker CL, Zou KH, Su J. Risk assessment of readmissions following an initial COPD-related hospitalization. Int J Chron Obstruct Pulmon Dis 2013;8:551-559.

8. Betancourt JR, Tan-McGrory A, Kenst KS. Guide to Preventing Readmissions among Racially and Ethnically Diverse Medicare Beneficiaries. Prepared by the Disparities Solutions Center, Mongan Institute for Health Policy at Massachusetts General Hospital. Baltimore, MD: Centers for Medicare \& Medicaid Services Office of Minority Health; September 2015.

9. Yale New Haven Health Services Corporation/Center for Outcomes Research \& Evaluation (YNHHSC/CORE). 2017 All-Cause Hospital-Wide Measure Updates and Specifications Report Hospital-Level 30-Day Risk-Standardized Readmission Measure - Version 6.0. Prepared for Centers for Medicare \& Medicaid Services (CMS); March 2017. Available at https://medicine.yale.edu/core

10. Elixhauser A, Steiner C, Harris DR, Coffey RM. Comorbidity measures for use with administrative data. Med Care 1998;36(1):8-27.

11. Ford ES, Croft JB, Mannino DM, Wheaton AG, Zhang X, Giles WH. COPD surveillance-United States, 1999-2011. Chest 2013;144(1): 284-305.
12. Arnett MJ, Thorpe RJ Jr, Gaskin DJ, Bowie JV, LaVeist TA. Race, medical mistrust, and segregation in primary care as usual source of care: findings from the Exploring Health Disparities in Integrated Communities Study. J Urban Health 2016;93(3):456-467.

13. Eisner MD, Blanc PD, Omachi TA, et al. Socioeconomic status, race and COPD health outcomes. J Epidemiol Community Health 2011; 65(1):26-34.

14. Bruse S, Sood A, Petersen H, et al. New Mexican Hispanic smokers have lower odds of chronic obstructive pulmonary disease and less decline in lung function than non-Hispanic whites. Am J Respir Crit Care Med 2011;184(11):1254-1260.

15. Miller MR, Crapo R, Hankinson J, Brusasco V, Burgos F, Casaburi $\mathrm{R}$, et al. General considerations for lung function testing. Eur Respir J 2005;26(1):153-161.

16. Sharma G, Kuo YF, Freeman JL, Zhang DD, Goodwin JS. Outpatient follow-up visit and 30-day emergency department visit and readmission in patients hospitalized for chronic obstructive pulmonary disease. Arch Intern Med 2010;170(18):1664-1670.

17. Rosa UW. Impact of cultural competence on medical care: where are we today? Clin Chest Med 2006;27(3):395-399.

18. Singh GK, Daus GP, Allender M, et al. Social determinants of health in the united states: addressing major health inequality trends for the nation, 1935-2016. Int J MCH AIDS 2017;6(2):139-164.

19. Goto T, Faridi MK, Gibo K, Camargo CA Jr, Hasegawa K. Sex and racial/ethnic differences in the reason for 30-day readmission after COPD hospitalization. Respir Med 2017;131:6-10.

20. Shaya FT, Maneval MS, Gbarayor CM, Sohn K, Dalal AA, Du D, et al. Burden of COPD, asthma, and concomitant COPD and asthma among adults: racial disparities in a medicaid population. Chest 2009; 136(2):405-411.

21. Weiss CO, Gonzalez HM, Kabeto MU, Langa KM. Differences in amount of informal care received by non-Hispanic whites and Latinos in a nationally representative sample of older Americans. J Am Geriatr Soc 2005;53(1):146-151.

22. Werre ND, Boucher EL, Beachey WD. Comparison of therapistdirected and physician-directed respiratory care in COPD subjects with acute pneumonia. Respir Care 2015;60(2):151-154. 